\title{
Rhodopsin gene copies in Japanese eel originated in a teleost-specific genome duplication
}

Yoji Nakamura ${ }^{1 *}$ D, Motoshige Yasuike ${ }^{1}$, Miyuki Mekuchi ${ }^{1}$, Yuki Iwasaki $^{1,2}$, Nobuhiko Ojima $^{1,3}$, Atushi Fujiwara ${ }^{1}$, Seinen Chow ${ }^{1}$ and Kenji Saitoh ${ }^{1,4}$

\begin{abstract}
Background: Gene duplication is considered important to increasing the genetic diversity in animals. In fish, visual pigment genes are often independently duplicated, and the evolutionary significance of such duplications has long been of interest. Eels have two rhodopsin genes (rho), one of which (freshwater type, fw-rho) functions in freshwater and the other (deep-sea type, ds-rho) in marine environments. Hence, switching of rho expression in retinal cells is tightly linked with eels' unique life cycle, in which they migrate from rivers or lakes to the sea. These rho genes are apparently paralogous, but the timing of their duplication is unclear due to the deep-branching phylogeny. The aim of the present study is to elucidate the evolutionary origin of the two rho copies in eels using comparative genomics methods.
\end{abstract}

Results: In the present study, we sequenced the genome of Japanese eel Anguilla japonica and reconstructed two regions containing rho by de novo assembly. We found a single corresponding region in a non-teleostean primitive ray-finned fish (spotted gar) and two regions in a primitive teleost (Asian arowana). The order of $d s$-rho and the neighboring genes was highly conserved among the three species. With respect to fw-rho, which was lost in Asian arowana, the neighboring genes were also syntenic between Japanese eel and Asian arowana. In particular, the pattern of gene losses in ds-rho and fw-rho regions was the same as that in Asian arowana, and no discrepancy was found in any of the teleost genomes examined. Phylogenetic analysis supports mutual monophyly of these two teleostean synteny groups, which correspond to the $d s$-rho and fw-rho regions.

Conclusions: Syntenic and phylogenetic analyses suggest that the duplication of rhodopsin gene in Japanese eel predated the divergence of eel (Elopomorpha) and arowana (Osteoglossomorpha). Thus, based on the principle of parsimony, it is most likely that the rhodopsin paralogs were generated through a whole genome duplication in the ancestor of teleosts, and have remained till the present in eels with distinct functional roles. Our result indicates, for the first time, that teleost-specific genome duplication may have contributed to a gene innovation involved in eel-specific migratory life cycle.

Keywords: Whole genome duplication, Teleostei, Anguilla, Rhodopsin paralogs, Visual adaptation, Phylogenomics, Synteny, Gene loss

\footnotetext{
* Correspondence: yojnakam@affrc.go.jp

${ }^{1}$ Research Center for Bioinformatics and Biosciences, National Research

Institute of Fisheries Science, Japan Fisheries Research and Education

Agency, 2-12-4 Fukuura, Kanazawa, Yokohama, Kanagawa 236-8648, Japan

Full list of author information is available at the end of the article
} 


\section{Background}

In the molecular evolution of organisms, gene duplication plays a pivotal role in preparing raw materials for evolution $[1,2]$. Whereas mutations in single-copy genes are under strong negative selection pressure, redundant gene copies enable a wider spectrum of mutations mostly deleterious. Moreover, some mutations may contribute to sub- or neo-functionalization of copies, making creative evolutionary changes. Gene duplication can occur at the single gene, segmental or chromosomal level, and even whole genome duplications are possible, which may cause the largest scale of divergence of gene functions. In particular, two rounds of whole genome duplication events at initial stages of vertebrate evolution have been important topics for several decades [2-5]. In fish, the ancestor of teleosts (infraclass Teleostei) underwent an additional round of genome duplication (TSD; teleost-specific genome duplication) around 300 million years ago [6-8], which has also attracted the attention of evolutionary biologists. About $48 \%$ of extant vertebrate species are teleosts $[9,10]$, and these live in highly diverse aquatic environments; from tropical coral reefs or rain forests to limit-cooled water under ice in polar regions or highland glacial lakes, from shallow estuaries to abyssal ocean trenches or far offshore open ocean surface, from highly alkalic to acidic waters, and from open waters under the sun to deep into ever dark caves. Thus, the correlation of whole genome duplication and eco-physiological diversification in the teleost lineage has been a topic that has attracted both interest and controversy in evolutionary biology [11-13].

Sensory organs are indispensable for most organisms, of which vision is specifically important for agile animals such as fish. Because of the differential penetration of lights of different wave lengths, diverse light sensitivity may be observed among fish species inhabiting different environments [14]. Vision also physiologically and ontogenetically changes: for example, fish visual sensitivity changes according to habitats shift from freshwater to marine environments $[15,16]$ and to food shift from carotenoid rich crustacean plankton to blue-green colored pelagic fish $[17,18]$. Diversification in light sensitivity at various wave lengths is achieved by evolutionary tuning and differential expression of visual pigment genes. Visual pigment genes have been subjected to studies of gene duplication. In general, fish species have five types of visual pigment genes, which enable effective perception of colors (ultraviolet, blue, green, and red) and dimlight. These subtypes are considered to have arisen in two rounds of whole genome duplications in the ancestor of vertebrates [19]. Regarding TSD, however, the signature remains obscure; one of the two duplicate copies of visual pigment genes that emerged as the result of TSD has been lost in most teleosts. Rather, within each of the five types, the visual pigment gene is often duplicated at the single gene level independently of the teleost lineages $[20,21]$. Rhodopsin is the visual pigment protein working for dim light vision [22], and the gene structure has been determined in many animals. The encoding gene (rho) is universally intronless in teleosts, which is believed to be due to a reverse-transcriptional insertion of the spliced messenger RNA transcribed at a distant locus early in the ray-finned fish lineage [23]. The original gene with introns before the insertion is utilized as retinal rhodopsin in tetrapods, but as exorhodopsin in fish [24]. It has been reported that rho is retained in the single copy state in most teleosts [25], but a few exceptional species with duplicate rho are scattered among teleostean tree of life, such as zebrafish, pearl eye, conger, and eel [26-29].

Freshwater eels of the genus Anguilla are among the few catadromous migrating teleost fish, which grow in freshwater areas and descend to the sea for reproduction. Majority of teleostean species live in either marine (55.5\%) or freshwater $(42.7 \%)$ environments, and the other small fraction (1.8\%) makes a round trip between the sea and freshwaters for growth and reproduction (diadromy) [30]. Catadromous fish comprise a further small fraction of diadromous fish. The majority of diadromous fish, such as salmons and freshwater gobies, spawn in freshwaters and grow in either marine or freshwater habitats (anadromous or amphidromous). The spawning area of Japanese eel Anguilla japonica Temminck \& Schlegel, 1846 has been determined to be located at the southern part of the West Mariana Ridge [31, 32]. After years of living in the freshwater or near-shore habitats in the northeast Asia, Japanese eels metamorphose into silver stage at onset of early maturation and start oceanic migration to reach the above-mentioned open ocean spawning area. Biologging and tracking experiments have revealed that the freshwater eels perform diel vertical migration between upper and lower mesopelagic zones (c.a. 200-1000 m) during oceanic migration (see [33], and references therein). The life cycle of the freshwater eels is thus distinct from other teleosts, which might be associated with their phylogenetic position: the superorder Elopomorpha, as well as Osteoglossomorpha, is considered to have diverged from basal teleosts (Fig. 1) [34, 35].

Ambient light condition of the migrating eels should then change according to their life stages. Carlisle and Denton [16] reported that the wavelength of maximum absorption in visual pigments was different between eels caught in the river and the sea, and further studies have shown that this trait is due to the expression switching of two rho genes in retinal cells [29, 36-38]. Origin of these rho, namely fw-rho for freshwater and $d s$-rho for deep-sea in this study, is clearly the result of a gene duplication event [29], but when the event occurred 


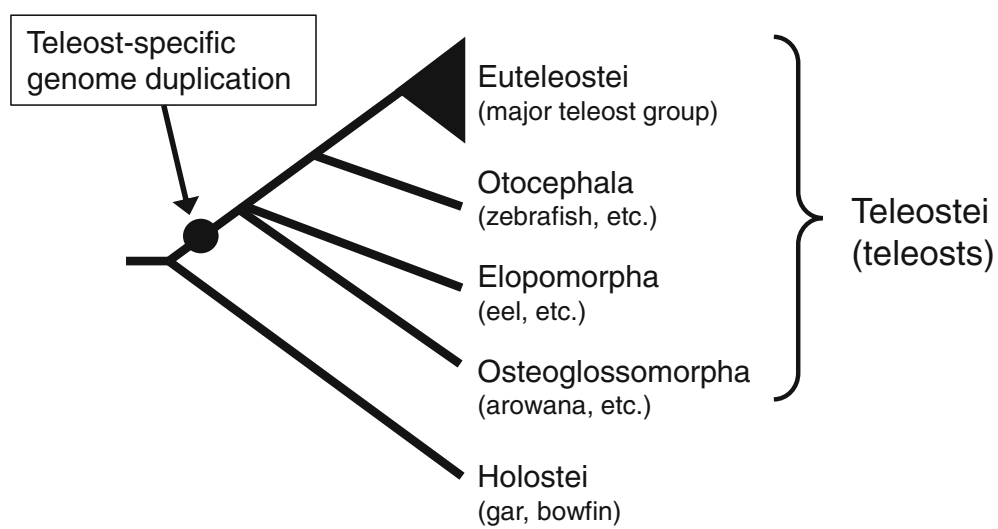

Fig. 1 Phylogenetic relationship among the fish lineages examined in this studyThe timing of teleost-specific genome duplication (TSD) is shown by a closed dot.

remains unsolved. From the deep-branching phylogeny of rhodopsin and related genes in fish, the duplicate genes might be derivatives of TSD, but previous studies have not yielded a clear conclusion [19, 39, 40]. The timing of TSD is estimated to predate the occurrences of Elopomorpha and Osteoglossomorpha, and postdate the divergence between Teleostei and Holostei (gar and bowfin) (Fig. 1). Actually, spotted gar, Lepisosteus oculatus, possesses a single copy of intronless rho in a genomic region on linkage group 5 (LG5), which suggests that this species may have retained the ancestral structure of rho region before TSD [19]. With reference to Osteoglossomorpha, the genome of Asian arowana, Scleropages formosus, was recently sequenced [41], but the structure of its rho region has not been fully investigated.

In this study, we compared eel's rhodopsin paralogs with those of other teleosts and vertebrates, such as Asian arowana and spotted gar, at the genomic level including synteny organization. The relationship between the evolutionary pathway of rhodopsin genes and migratory ecology is discussed.

\section{Methods}

\section{Genome sequencing and assembly}

All experiments were conducted following principles and procedures approved by the guidelines for the care and use of live fish at National Research Institute of Fisheries Science. First, an adult individual of Japanese eel, which was of full-life cycle culture (F2 generation derived from wild-caught grandparents) in National Research Institute of Aquaculture [42], was anesthetized with $0.2 \% 2$ phenoxyethanol (Wako, Osaka, Japan) prior to sampling, and the genomic DNAs were then extracted from whole blood following the previously reported method [43]. Preparation of sequence templates for 454 FLX+ and Illumina GAII or HiSeq followed the manufacturers' instructions. We first assembled 454 genomic shotgun and paired-end reads of $3 \mathrm{~kb}$ and $6 \mathrm{~kb}$ by Newbler assembler (version 2.9; Roche Diagnostics). Then the Illumina paired-end reads, mate-pair reads (75 or $100 \mathrm{bp} \times 2$ with various insert lengths) and single reads (75 bp) spun off from quality screening of read pairs worked for improvement of sequence accuracy mapping them by bowtie 2 (version 2.1.0) onto the scaffolds made up of 454 reads allowing one base indels [44]. As a result, 591,560 sites were overridden by sequences called by Illumina. The improved 454 scaffolds and contigs were then bridged by Illumina paired-end and mate-pair reads with SSPACEbasic (version 2.0) [45]. Bridging process was progressive in which paired-end (800 bp), mate-pair reads of $5 \mathrm{~kb}$, $8 \mathrm{~kb}, 10 \mathrm{~kb}$, and finally $15 \mathrm{~kb}$ worked sequentially (Additional file 1). Gaps in the scaffolds were filled by the Illumina reads with GapFiller (version 1.10) [46]. Total read coverage of the assembly was $129 \times$ the expected genome size [47]. The Illumina paired-end reads (800 bp) were used also for genome size estimation based on $k$-mer frequency by JELLYFISH [48]. The scaffold sequences are deposited to DDBJ/GenBank/EMBL databases under accession numbers BDQN01000001-BDQN01195366. The linkage marker sequences obtained in the previous study [49] were mapped to the scaffold sequences using BLASTN (identity > $=90 \%$ ). The markers matched to two or more scaffolds were excluded, and the scaffolds with single-hit markers were attributed to the linkage groups (LG1 to LG19). The correspondences to LGs were further manually checked, and the scaffolds attributed to two or more LGs were split into consistent ones based on the locations of marker. Scaffolds $>=2000 \mathrm{bp}$ in length were used for subsequent analysis.

\section{RNA preparation and sequencing}

From the same individual of Japanese eel as used for whole genome sequencing, brain, gill, esophagus, stomach, 
anterior intestine, posterior intestine, rectum, pancreas, liver, spleen, gall bladder, swim bladder, muscle, head kidney, body kidney, urinary bladder, blood, and skin were dissected out. All organs were immediately immersed in RNAlater stabilized solution (Thermo Fisher Scientific, Waltham, MA). Total RNA was extracted using the RNeasy Lipid Tissue Mini Kit (Qiagen GmbH, Hilden, Germany), according to the manufacturer's protocol. RNA quality was evaluated based on the proportion of rRNA using an Agilent 2100 Bioanalyzer RNA 6000 Nano Kit (Agilent Technologies, Palo Alto, CA, USA). A complementally DNA libraries were constructed, followed by DSN Normalization using Duplex-Specific thermostable nuclease. Libraries were sequenced by Illumina HiSeq 2000 platform equipped with $100 \mathrm{bp}$ paired-end module. After sequencing, the raw reads were filtered by removing low-quality reads $(\mathrm{QV}<20)$.

\section{Gene prediction}

The protein-coding genes in the Japanese eel genome were predicted using AUGUSTUS (version 3.2.2) [50]. First, RNA-seq reads of Japanese eel sequenced in this study were mapped to the scaffolds by TopHat [51] and assembled by Cufflinks [52]. In addition, protein sequences of 11 fish species: spotted gar (Lepisosteus oculatus), Mexican tetra (Astyanax mexicanus), zebrafish (Danio rerio), Atlantic cod (Gadus morhua), Nile tilapia (Oreochromis niloticus), platyfish (Xiphophorus maculatus), Amazon molly (Poecilia formosa), medaka (Oryzias latipes), stickleback (Gasterosteus aculeatus), greenpuffer (Tetraodon nigroviridis), and fugu (Takifugu rubripes), were downloaded from the ENSEMBL database (Release 84) [53], and mapped to the Japanese eel scaffolds by TBLASTN with E-value $<10^{-4}$. Next, the scaffold sequences were scanned by the generic model in AUGUSTUS, using the map information of RNA-seq and ENSEMBL protein data as hints, and a total of 85,987 genes were predicted. Then, the predicted gene sequences were compared to the reference full-length protein sequences extracted from four fish genome data of ENSEMBL (zebrafish, medaka, greenpuffer, and fugu) by BLASTP [54] with E-value $<10^{-10}$, and a total of 1776 sequences were selected as well-validated ones whose lengths were close to those of the reference sequences (difference $<5 \%$ ). Using these sequences, the training model of eel genes was constructed, and the gene prediction was performed by AUGUSTUS again based on the above transcript and protein hints. Finally, InterProScan [55] was performed to the predicted gene sequences, and those matched by any domain or supported by any AUGUSTUS hint were collected as valid protein-coding genes.

\section{Synteny and phylogenetic analyses}

The above-mentioned ENSEMBL data of 11 fish species were used for ortholog and synteny comparison. In addition, as outgroups in phylogenetic analysis, the gene sequences of chicken (Gallus gallus), and human (Homo sapiens) were also downloaded from the ENSEMBL. Furthermore, the genome data of two fish species, Asian arowana (Scleropages formosus) and northern pike (Esox lucius) [56], were downloaded from the GenBank (accession number: GCF_001624265) and the online resource at the University of Victoria (http://web.uvic.ca/grasp/ pike/), respectively. From the transcriptome data of northern pike, protein-coding sequences were predicted by TransDecoder [57]. Orthologous genes among Japanese eel and these 15 species (13 fish species, chicken and human) were first estimated by OrthoMCL [58], and the genes missed by AUGUSTUS prediction or in the database annotation were further predicted by Exonerate [59] with protein identity $>40 \%$ based on the protein sequences of Asian arowana and spotted gar. As a close relative of Japanese eel, the genome data of European eel (Anguilla anguilla) [60] was downloaded from the GenBank (accession number: GCA_000695075), and alignments of the scaffold sequences between these eels were constructed by MUMmer 3 [61].

For phylogenetic analysis, we selected species with good recovery of two syntenic regions in which Japanese eel retains rho paralogs (rho regions). Mexican tetra and platyfish were not included in further analysis because of disjuncture in these rho regions. Amino acid sequences were aligned with MAFFT ver. 6.7 [62] with manual adjustment. We pruned sites with gaps in more than a half of OTUs. DNA sequence alignments were deduced from the amino acid alignments. Nucleotides at fast evolving 3rd codon positions were treated in three ways for phylogenetic analysis: included in the analysis without modification, RY-coding [63] for accounting translations only, and excluded from the analysis. Substitution models were compared with MEGA ver. 6 [64]. We conducted phylogenetic analysis both on alignments of each separate gene sequences and on concatenated supermatrix sequences of the rho region. Maximum likelihood (ML) trees were inferred with PAML ver. 4.9 [65] by repeated local rearrangements [66]. Tree robustness was assessed by RELL method [67] with CONSEL ver. 0.2 [68]. Source of tree incongruence especially on position of the eel fw-rho was tested excluding sites around those showing higher non-synonymous substitutions detected with JCoda ver. 1.4 [69].

\section{Results}

Japanese eel genome and rho loci

We obtained a total of 20,564 scaffolds with $1055 \mathrm{Mb}$, accounting for $98.9 \%$ of the Japanese eel genome [47] 
(Table 1), while the genome size based on $k$-mer frequency was estimated to be $920 \mathrm{Mb}$ (Additional file 2). Of these, 868 scaffolds totaling $529 \mathrm{Mb}$ were attributed to each of 19 linkage groups of Japanese eel (Additional file 3). In 20,564 scaffolds, we predicted a total of 26,689 protein-coding genes, and from these detected two intronless rhodopsin genes, $d s$-rho and fw-rho (Additional file 4). These genes were located on two scaffolds, namely scaffold $3(5,957,805 \mathrm{bp}$ in total) on LG12 and scaffold 435 (438,394 bp in total) with no attribution to LG, respectively. In scaffold 3, a total of 127 genes including $d s$-rho were predicted. Since scaffold 435 was not fully assembled compared to scaffold 3 , only eight genes including $f w$-rho were detected.

Next, using these gene sequences in Japanese eel, we searched for orthologous genes in primitive ray-finned fish genomes, such as those of spotted gar and Asian arowana. We found that many of the genes in the Japanese eel scaffold 3 were orthologous to those encoded in LG5 of spotted gar. Particularly, the region of approximately $3 \mathrm{Mb}$ surrounding ds-rho in Japanese eel had a large synteny to the rho region in LG5 of spotted gar (Fig. 2). Of 138 genes encoded in $20-29 \mathrm{Mb}$ region of spotted gar's LG5, 67 genes were detected in the $d s$-rho region of Japanese eel. The 57 genes were in the same transcriptional direction as those of spotted gar, and the other 10 genes were likely to be due to small inversions or translocations. We found that the Asian arowana genome also had two scaffolds syntenic to the rho region of spotted gar, namely scaffold 133 (6,725,481 bp in total) attributed to chromosome 11 and scaffold $11(8,087,411$ bp in total) attributed to chromosome 3, respectively (Fig. 2). In scaffold 133, 81 of 138 genes in spotted gar's LG5 (20-29 Mb) were detected as orthologs, and 57 genes were common to those of the $d s$ rho region in Japanese eel. In scaffold 11, 28 of the spotted gar's 138 genes were detected, but rho was absent.

The microsyntenies neighboring rho were further compared adding species of Otocephala (Mexican tetra and zebrafish) and Euteleostei (northern pike, Atlantic cod, Nile tilapia, platyfish, Amazon molly, medaka, stickleback, greenpuffer, and fugu). We focused on 11 genes including rho in the spotted gar LG5 as a

Table 1 Assembly statistics of the Japanese eel genome

\begin{tabular}{ll}
\hline No. of scaffolds & 20,564 \\
Total base pairs (Mb) & 1055 \\
Average scaffold size (kb) & 51.3 \\
No. of scaffolds mapped to LGs & 868 \\
Total base pairs mapped to LGs (Mb) & 529 \\
Predicted protein-coding genes* & 26,689 \\
\hline
\end{tabular}

*Predicted by AUGUSTUS and InterProScan. reference, namely lrig1, slc25a26, magi1, rho, adamts9, prickle2, pphln1, slc2a9l1, psmd6, atxn7, and thoc7 (Fig. 3). The order of 10 genes, except for $p p h \ln 1$, were perfectly conserved between Japanese eel's scaffold 3 carrying ds-rho and spotted gar's LG5. In addition, we found that the gene order in scaffold 133 of Asian arowana was identical to that in Japanese eel's scaffold 3. Regarding fw-rho region, five genes (magi1, rho, prickle2, $p p h \ln 1$, and $a t x n 7)$ were conserved between Japanese eel (scaffold 435) and spotted gar, but the other six were absent. To be precise, atxn7 was predicted in the end of scaffold 435 and the absence of thoc7 is unclear, but thoc7 was not predicted in any other scaffolds. In scaffold 11 of Asian arowana, four (magi1, prickle2, pphln1, and $\operatorname{atxn7)}$ out of 11 genes were found. Therefore, except for absence of rho, this region of Asian arowana was syntenic to scaffold 435 of Japanese eel. For simplicity, we refer to these putatively orthologous syntenies of the rho region as \#1 (eel scaffold 3 and arowana 133) and \#2 (eel scaffold 435 and arowana 11). Regarding otocephalan and euteleostean species, the syntenic regions were also found in all the genomes examined, although the sequences were fragmented or some genes were not found (Fig. 3, and Additional files 5 and 6). All the cases were explainable by deletions of single gene in either of these syntenies. Pattern of the gene loss of rho paralogs was contrasting between Asian arowana and euteleosts examined.

Finally, we examined rho regions in the European eel genome. The scaffolds of European eel were more fragmented than those of Japanese eel, and the $d s$-rho region was split into two scaffolds in European eel (accession numbers: AZBK01S000177 and AZBK01S000303), but the gene order was eventually the same as Japanese eel (Additional file 7, Figs. S3a, b). Regarding fw-rho region, the scaffold of European eel (accession number: AZBK01S000274) was longer than that of Japanese eel (scaffold 435), whereby scaffold 435 was linked to a scaffold attributed to LG6 (scaffold 195,311) based on sequence alignment (Additional file 7, Fig. S3c).

\section{Phylogenetic analysis}

Of eight genes commonly found in the rho region among vertebrates (Additional file 6), Japanese eel retains two copies of magi1, rho, prickle 2 and atxn7 on two syntenies. We thus employed these four genes for phylogenetic analysis. Best fit models for phylogenetic analysis implemented in PAML were mostly JTT with gamma correction for amino acid alignments and GTR or TN93 with gamma correction for DNA alignments (Additional file 8). ML analysis of interrelationships among rho orthologs and paralogs gave skewed tree topologies (Figs. 4a, b, and Additional file 9). Two copies from Japanese eel got together in a clade independent 

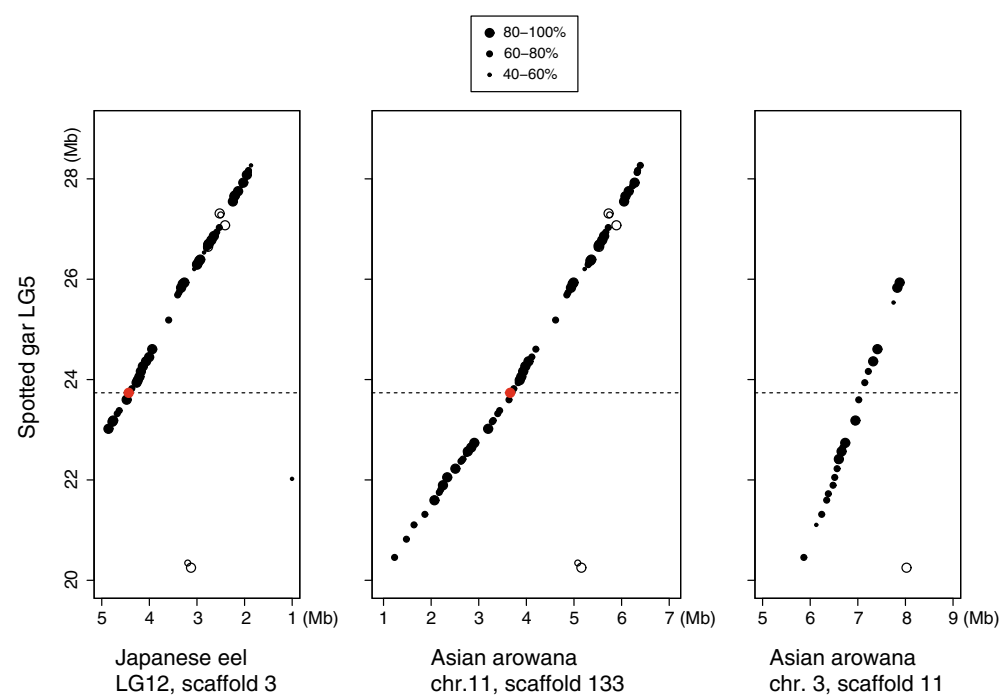

Fig. 2 Dot plot for macrosynteny of rho regionFor Japanese eel scaffold 3, and Asian arowana scaffolds 133 and 11, the positions of genes matched to those in spotted gar's LG5 region (20-29 Mb) are plotted. Each of the dots is the midpoint of predicted start and end positions of gene, and the size indicates the degree of amino acid identity (\%) to the ortholog in spotted gar; in the same transcriptional direction (closed dot) or opposite direction (open dot). The rhodopsin gene is colored in red and the position in spotted gar's LG5 is shown in dashed line.

from most of the others as was presented in previous studies [40, 70]. In addition, some disagreements in topology were observed among different sets of sequence alignment. Branch support values were low (81 and $84 \%$ on averages). Excluding sites around those with higher non-synonymous substitutions (Additional file 10) did not resolve this tree skewness (Additional file 9). Trees given on $\operatorname{atxn} 7$ were also skewed (Additional file 9). Analysis of concatenated sequences, however, recovered two mutually monophyletic synteny clusters (Figs. 4c, d). Topologies given on different sequence datasets were mostly congruent with higher branch supports (both 93\% on averages). Clades of Clupeocephala (zebrafish and higher teleosts) and Euteleostei (northern pike and

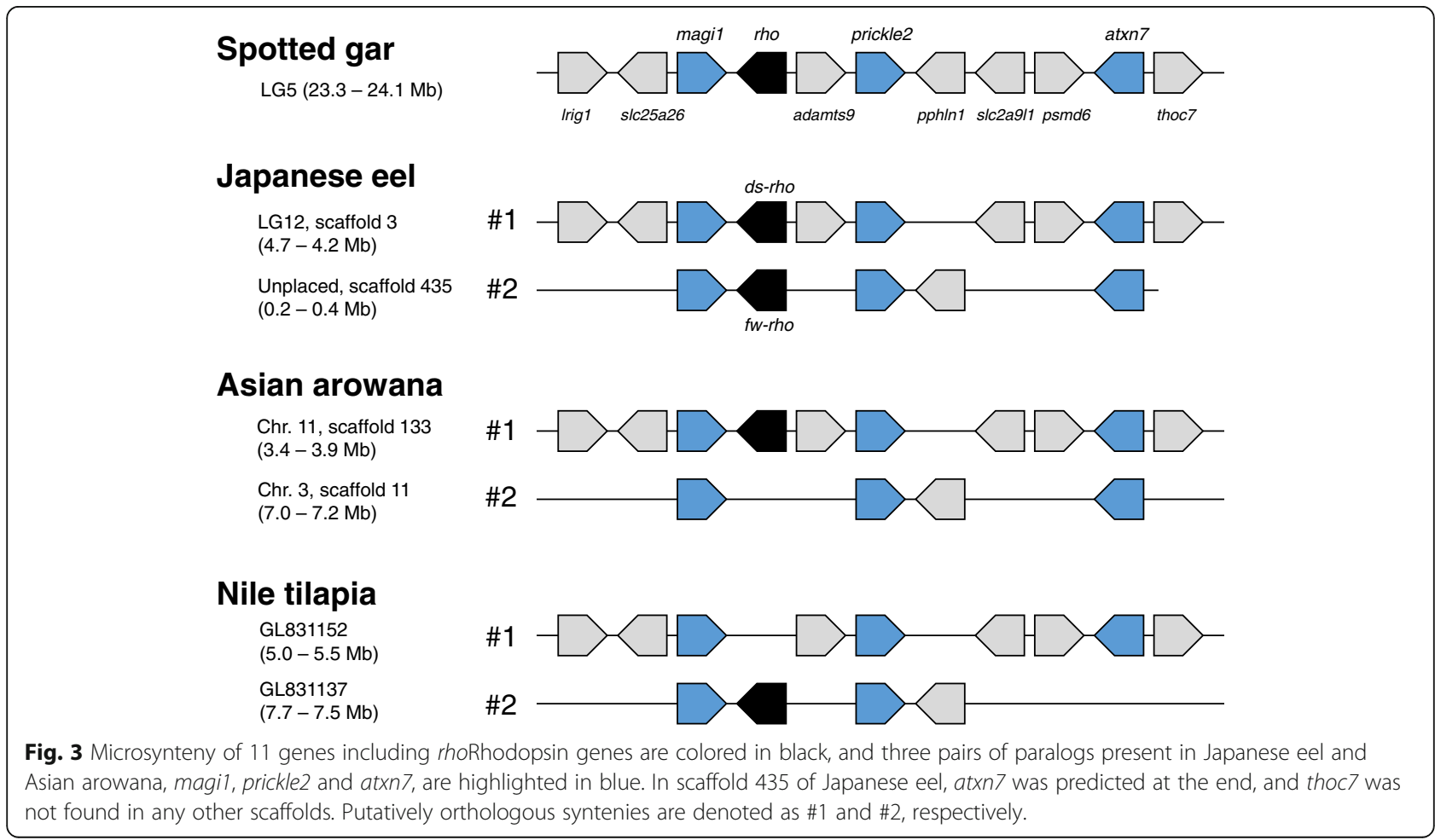




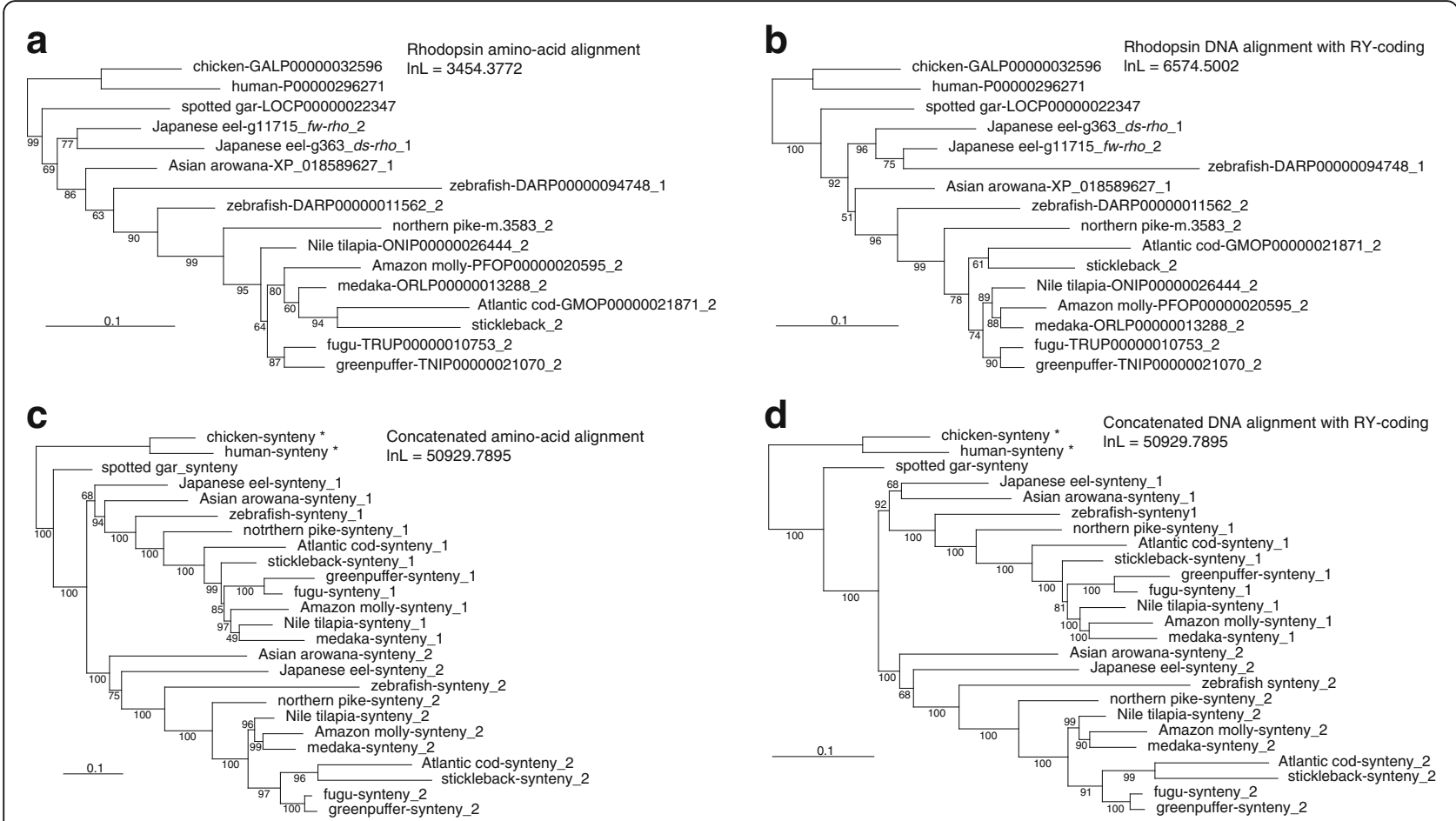

Fig. 4 Phylogenetic treesPhylogenetic relationships among $r$ ho $(\mathbf{a}, \mathbf{b})$ and concatenated synteny $(\mathbf{c}, \mathbf{d})$ sequences. Amino acid (a, $\mathbf{c})$ and RY-coded DNA $(\mathbf{b}, \mathbf{d})$ sequence-based trees are shown. Numbers attached to teleostean gene IDs denote syntenies on which genes are encoded. Numbers beside branches indicate local bootstrap [66] support (\%) of each branch. ${ }^{*}$ Chicken and human rho sequences of synteny data are from a remote locus.

higher) were recovered in each of two synteny clusters. Trees based on magi1 and prickle 2 showed good recovery of monophyletic synteny clusters (Additional file 9).

\section{Discussion}

We sequenced the genome of Japanese eel, mapped approximately half of the scaffolds assembled to linkage groups. Although the total size of scaffolds was highly consistent with the experimentally estimated genome size [47], it was $\sim 15 \%$ larger than the estimate of genome size based on $k$-mer frequency. Since the genomes sizes of eel species have been recently reconsidered as with their repetitive regions and heterozygous sites [71], further improvements should also be done for the Japanese eel genome. In the present study, we focused on rhodopsin genes in Japanese eel and reconstructed the two loci of $r h o$ from the scaffold sequences. The $d s-r h o$ and $f w$-rho were located on different scaffolds, the longer one covering $d s$-rho was nearly $6 \mathrm{Mb}$ in size, and the shorter one $(\sim 400 \mathrm{~kb})$ had at least three paralogs (magi1, prickle 2 and atxn7) next to fw-rho. We can thus conclude that the duplication of rho in Japanese eel was neither at the single gene level nor tandem, as in the case of other visual pigment genes, but on a larger scale, and that it was followed by losses of neighboring paralogs. In addition, the scaffold of $d s$-rho widely corresponded to the region of spotted gar's LG5, indicating that the genomic region surrounding $d s$-rho has kept the ancestral structure. Most importantly, we found that the Asian arowana genome had two scaffolds similar to those of $d s$-rho and fw-rho in Japanese eel, although a copy of rho was lost. Since the both scaffolds widely correspond to spotted gar's LG5 region, the Asian arowana rho regions are products of a large scale duplication. Eel (Elopomorpha) and arowana (Osteoglossomorpha) are both considered to have been diverged at the early stage of teleost evolution [72]. This suggests a likely scenario for the origin of two regions of rho in Japanese eel and Asian arowana: a large scale duplication occurred in the common ancestor of eel and arowana, and after the divergence of these lineages, both rho genes have been maintained in Japanese eel, whereas a copy of rho has been lost in Asian arowana. An alternative scenario is that large scale duplications have occurred separately in each lineage after the divergences of eel and arowana, respectively, and a copy of rho has been lost in Asian arowana. Based on parsimony, this second scenario is less likely, because it assumes two parallel duplication events. Moreover, it should be noted that TSD is estimated to have occurred shortly before the divergences of 
eel and arowana [34, 73]. Thus, the second scenario needs further assumptions of loss of either of TSDderived rho regions and recurrent parallel duplications. In the first scenario on the other hand, the observation can be simply explained by a single large-scale duplication before the divergences of eel and arowana and a loss of rho in the arowana lineage. Considering that the large scaffolds of Asian arowana were mapped on different chromosomes, it is reasonable to conclude that the large-scale duplication was TSD. In this study, we identified $d s$-rho of Japanese eel on the LG12 according to the mapped linkage markers. In addition, the comparison with the European eel genome suggested that $f w$-rho might be located on LG6. In the previous study, the LG12 and LG6 of Japanese eel or their backbone regions were estimated to have been built at TSD because of wide correspondences to spotted gar LG5 and medaka's chromosomes 5 and 7 [49]. Thus, it seems likely that the rho genes in eel have been retained in the descendant regions of TSD-derived chromosomes.

Scaffold 435 of fw-rho in Japanese eel is short and its correspondence to Asian arowana's scaffold 11 remains obscure, raising the additional possibility that fw-rho in Japanese eel has been generated by another duplication (e.g., lineage-specific segmental duplication). Although this third scenario also assumes the loss and a recurrent duplication of the rho region after TSD as in the second scenario, multiple losses and duplications may be possible on a smaller scale, such as the tandem duplications reported in other visual pigment genes. Therefore, we further examined the microsynteny around rho locus among Japanese eel and other teleosts. In spotted gar as an outgroup, rho was located within the region from lrig1 to thoc7 in LG5, and the synteny was also observed in the corresponding regions of Japanese eel and Asian arowana, strongly suggesting that the gene order is the ancestral form in teleosts. Considering that paralogs of magi1, prickle 2 and $a t x n 7$ are present in the fw-rho region of Japanese eel, at least eight genes (magi1, ds-rho, adamst9, prickle2, pphln1, slc2a9l1, psmd6, and atxn7) as a template should have been doubled in the duplication event, followed by losses of several paralogs. Apparently, this is also the case in Asian arowana. Here, it is important to note that the pattern of gene losses was the same between Japanese eel and Asian arowana, except for that a copy of rho is lost in Asian arowana. Many studies have shown that either of the paralogs generated by duplication will be rapidly lost, while a number of paralogs might diverge functionally to each other [74-76]. The pattern of gene losses is a good signature for estimating the evolutionary scenario of duplicate genomic regions [77], because the genes lost once in the template region would never be restored by duplication again. In this study, for example, we can say that the $f w$-rho region with $p p h \ln 1$ in
Japanese eel should have been generated before the pphln1 paralog was lost in the $d s$-rho region, and so too with those in Asian arowana. In particular, four genes, adamst 9 , pphln1, slc2a9l1, and psmd6, were lost in the same pattern between Japanese eel and Asian arowana. Assuming that the duplications occurred separately in each of eel and arowana lineages, these coincidences are unlikely. Moreover, in all the teleost genomes examined, we did not find any cases inconsistent with this pattern. Thus, these results suggest that the gene orders in two rho regions observed in Japanese eel had already emerged in the ancestor of eel, arowana, and clupeocephalan (Otocephala + Euteleostei) lineages (Fig. 5). Whereas zebrafish and Mexican tetra have two copies of rho, all the euteleosts examined have a single copy of rho, but the gene loss was in the opposite side from that in Asian arowana. This is also explained by our hypothesis that two rho genes were still present at the divergence of teleostean lineages. Thus, we propose that the losses of rho on opposite regions occurred independently in arowana and clupeocephalan lineages after their divergence; otherwise it would be difficult to explain the loss pattern of rho and adamst 9 paralogs between magi1 and prickle2. Regarding two rho copies of zebrafish, phylogenetic analysis has recently suggested that the duplication occurred early in a teleost lineage, and either of the paralogs has evolved under positive selection [39]. According to our hypothesis, considering that the zebrafish lineage had branched at the base of Clupeocephala, it is possible that two rho copies predating the divergence of teleostean lineages have remained till the present in zebrafish and lost in the euteleostean ancestor. This possibility may be further tested by comparison to two rho copies in Mexican tetra in the same superorder (Otophysi) as zebrafish.

Two synteny clusters of the rho region among 11 teleosts in trees based on concatenated sequences (Figs. 4c, d) were in accord with the first scenario, because mutual monophyly of these synteny clusters can be traced back to the teleostean root. Recovery of outline of the teleostean interrelationships (clupeocephalan and euteleostean clades) in both synteny clusters with high branch supports indicates that the trees obtained from concatenated sequences are close to the true tree. The results from concatenated sequences, however, do not necessarily support the first scenario, because the two paralogous rho regions are merely vehicles of the gene of interest. Clustering of the eel rho copies relative to other teleosts in trees solely based on rho sequences regardless of exclusion of sites with higher non-synonymous substitutions (Figs. 4a, $\mathrm{b}$ and Additional File 10) is possible upon delayed resolution of tetrasomy [78] after TSD. Tetrasomy of chromosomal segments homogenizes homeolog sequences through recombination. Phylogenetic affinity of eel's two rho copies could arise, if the rho region was tetrasomic 


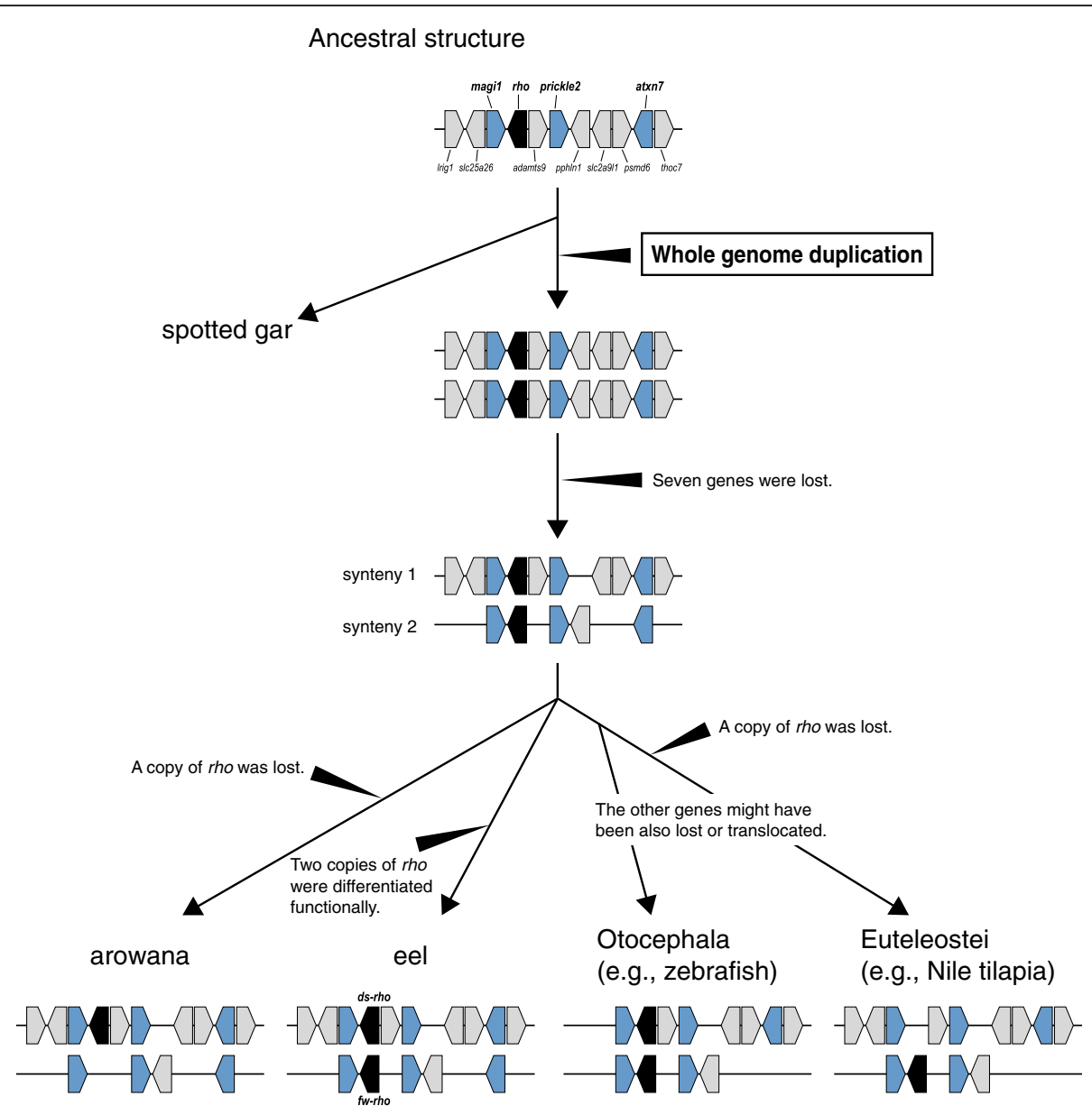

Fig. 5 Evolutionary scheme of rho region in teleostsRhodopsin genes are colored in black, and three pairs of paralogs present in Japanese eel and Asian arowana, magi1, prickle2 and atxn7, are highlighted in blue.

and the resolution (evolution of paralogy) was delayed particularly in the eel lineage. Atxn7-based trees also show clusters of eel and/or arowana paralogs (Additional file 9). Allotetraploidy (no tetrasomy predicted) at TSD is suggested [79], but tetrasomy can occur even after allotetraploidization [80]. Another possibility for this skewed tree shape arises upon the second scenario with recurrent gene losses/duplications.

Nevertheless, dense taxon sampling of rho sequences [39], magi1 and prickle2 sequences in our study (Additional file 9) yielded similar results with those based on concatenated sequences. Differences of phylogenetic resolving powers in different sizes of sequence datasets (either or both dimensions of length and number of OTUs) would be a source of the incongruences. Our results suggest affinity of $d s$-rho and $f w$-rho with synteny 1 and 2 clusters respectively, and thus the origin of $d s$ - and fw-rho genes can be traced back to the basal teleostean divergence near TSD. Thus, phylogenetic analysis supports the hypothesis that rho paralogs in Japanese eel have originated from TSD.
Finally, our results might shed light on the relationship between TSD and evolution of elopomorph species. Regarding sexual maturation, hormone receptor paralogs built at TSD, which are present in most other teleosts, have been examined also in eels [77, 81-83]. Moreover, genome-wide studies have shown that eels have the complete set of Hox gene clusters doubled at TSD [60, 84], while other teleosts lack some of the genes. Although such duplicate genes are likely involved in eels' life cycle, the genes are largely conserved among teleosts and might not be directly correlated with eels' unique adaptation to the freshwater and marine environments. Since the two rhodopsins in eel have been tuned for these environments, respectively [29,36-38], our finding provides the first evidence about the functional differentiation of TSD-derived paralogs with special reference to the eel-specific ecological traits. The species which had deviated earlier in elopomorph divergence are distributed from estuary (e.g., tarpon) to deep sea (e.g., gissu). After their divergence, the lineages of eels and relatives (conger, gulper eel, etc.) are inferred to have diverged 
$[73,85,86]$. These studies imply that the aquatic habitats of elopomorph species have been often changed. Therefore, the present study raises the interest in the correlation between such a habitat alteration and the functions of rhodopsin in elopomorph species. For example, whether two copies of rho are maintained or either is lost in these species would be a simple but important topic. Japanese Conger conger myriaster has two copies of rho orthologous to those of Japanese eel, respectively [28], hence these genes are also derived from TSD as shown in this study. Previous studies showed functional differentiation of these rho copies in Japanese conger but in a different way from Japanese eel $[28,70,87]$, suggesting multiple occurrence of the functional differentiation of rho paralogs by minor mutations in elopomorph fish retaining paralogs in their genome. In Anguilla, such as Japanese eel and European eel, the ancestral forms of rho may have been both deep-sea types, one of which has been mutated toward a freshwater-type, because a recent study suggested that these species evolved from a deep-sea habitat [88]. Thus, maintenance and sub-functionalization of the rho paralogs might be correlated with the wide distribution of elopomorph species from freshwater to the deep sea. It is difficult to answer in this study due to lack of other genome data on how often the functional changes of rho paralogs occurred in Elopomorpha. However, it should be stressed that maintenance of TSD-derived rho paralogs may have finally contributed to the establishment of eel's life cycle, particularly the habitat alteration by longdistance migration between distinct aquatic environments. Further research based on genome-wide comparison may reveal whether or not this is a rare case with regard to the evolutionary impact of TSD.

\section{Conclusions}

Two copies of eel rhodopsin gene were generated most likely at TSD. This is the first finding that TSD $(\sim 300$ million years ago) has remotely lead to the gene innovation involved in eel-specific migratory life cycle. Further researches may provide insight into correlations between the functional differentiation of TSD-derived paralogs and the diversification of elopomorph species.

\section{Additional files}

Additional file 1: Table S1. Sequencing results of the Japanese eel genome and cDNA. (XLS $32 \mathrm{~kb}$ )

Additional file 2: Figure S1. $K$-mer analysis using the Illumina pairedend reads of Japanese eel. (PPTX $42 \mathrm{~kb}$ )

Additional file 3: Table S2. LG mapping of the Japanese eel scaffolds. (XLS $335 \mathrm{~kb}$ )

Additional file 4: Table S3. Annotation of protein-coding genes predicted in the Japanese eel genome. (XLS $6125 \mathrm{~kb}$ )
Additional file 5: Figure S2. Microsynteny of rho region in other teleost genomes. (EPS $1575 \mathrm{~kb}$ )

Additional file 6: Table S4. Conservation of genes (Irig 1 - thoc7) around rho among vertebrates. (XLSX $15 \mathrm{~kb}$ )

Additional file 7: Figure S3. Comparison of rho regions between Japanese eel and European eel. (PDF 638 kb)

Additional file 8: Table S5. Substitution models employed in ML phylogenetic tree search. (XLS 28 kb)

Additional file 9: Supplement trees. (TXT $19 \mathrm{~kb}$ )

Additional file 10: Figure S4. Non-synonymous vs. synonymous substitution (dN/dS) ratio along rho sequence alignment with 25 aa window sliding every 5 aa. (PNG 70 kb)

\section{Acknowledgments}

We thank Hideki Tanaka for providing Japanese eel specimen.

\section{Funding}

This study was supported by a grant-in-aid from the Fisheries Agency, Ministry of Agriculture, Forestry and Fisheries of Japan to the Japan Fisheries Research and Education Agency ("Molecular genetic research project for developing stock management and breeding technologies of the Japanese eel"). The funder had no role in the design of the study and collection, analysis, or interpretation of data or in the writing of the manuscript.

\section{Availability of data and materials}

The nucleotide sequences determined in this study were deposited to DDBJ/GenBank/EMBL under accession numbers BDQN01000001BDQN01195366.

\section{Authors' contributions}

$\mathrm{KS}$ and $\mathrm{YN}$ conceived of the study. MY, MM, NO, AF, and KS performed the experiments. YN, MY, YI, and KS analyzed the data. SC revised it critically for important intellectual content. YN, MY, MM, and KS wrote the manuscript. All authors read and approved the final manuscript.

Ethics approval and consent to participate

Not applicable.

\section{Consent for publication}

Not applicable.

Competing interests

The authors declare that they have no competing interests.

\section{Publisher's Note}

Springer Nature remains neutral with regard to jurisdictional claims in published maps and institutional affiliations.

\section{Author details}

${ }^{1}$ Research Center for Bioinformatics and Biosciences, National Research Institute of Fisheries Science, Japan Fisheries Research and Education

Agency, 2-12-4 Fukuura, Kanazawa, Yokohama, Kanagawa 236-8648, Japan. 2Present address: National Institute of Genetics, 1111 Yata, Mishima, Shizuoka 411-8540, Japan. ${ }^{3}$ Present address: Japan Fisheries Research and Education Agency, 2-3-3 Minatomirai, Nishi, Yokohama, Kanagawa 220-6115, Japan. ${ }^{4}$ Present address: Tohoku National Fisheries Research Institute, Japan Fisheries Research and Education Agency, 3-27-5 Shinhama, Shiogama, Miyagi 985-0001, Japan.

Received: 13 July 2017 Accepted: 11 October 2017

Published online: 17 October 2017

\section{References}

1. Graur D, Li W-H. Fundamentals of molecular evolution. 2nd ed. Sinauer: Sunderland, Ma; 2000.

2. Ohno S. Evolution by gene duplication. Berlin, New York: SpringerVerlag; 1970. 
3. Kuraku S, Meyer A, Kuratani S. Timing of genome duplications relative to the origin of the vertebrates: did cyclostomes diverge before or after? Mo Biol Evol. 2009;26(1):47-59.

4. Dehal P, Boore JL. Two rounds of whole genome duplication in the ancestral vertebrate. PLoS Biol. 2005;3(10):e314.

5. Holland PW, Garcia-Fernandez J, Williams NA, Sidow A. Gene duplications and the origins of vertebrate development. Dev Suppl. 1994:125-33.

6. Vandepoele K, De Vos W, Taylor JS, Meyer A, Van de Peer Y. Major events in the genome evolution of vertebrates: paranome age and size differ considerably between ray-finned fishes and land vertebrates. Proc Natl Acad Sci U S A. 2004;101(6):1638-43.

7. Taylor JS, Van de Peer Y, Braasch I, Meyer A. Comparative genomics provides evidence for an ancient genome duplication event in fish. Philos Trans R Soc Lond Ser B Biol Sci. 2001;356(1414):1661-79.

8. Wittbrodt J, Meyer A, Schartl M. More genes in fish? BioEssays. 1998; 20(6):511-5

9. Eschmeyer WN, Fong JD. Species of fishes by family/subfamily. In., march 1. EDN. 2017;

10. The IUCN Red List of Threatened Species, Version 2016-3 [http://www. iucnredlist.org].

11. Clarke JT, Lloyd GT, Friedman M. Little evidence for enhanced phenotypic evolution in early teleosts relative to their living fossil sister group. Proc Natl Acad Sci U S A. 2016:113(41):11531-6.

12. Glasauer SM, Neuhauss SC. Whole-genome duplication in teleost fishes and its evolutionary consequences. Mol Gen Genomics. 2014;289(6):1045-60.

13. Hoegg S, Brinkmann H, Taylor JS, Meyer A. Phylogenetic timing of the fishspecific genome duplication correlates with the diversification of teleost fish. J Mol Evol. 2004;59(2):190-203.

14. Crescitelli F, McFall-Ngai M, Horwitz J. The visual pigment sensitivity hypothesis: further evidence from fishes of varying habitats. J Comp Physiol A. 1985;157(3):323-33.

15. Temple SE, Veldhoen KM, Phelan JT, Veldhoen NJ, Hawryshyn CW. Ontogenetic changes in photoreceptor opsin gene expression in coho salmon (Oncorhynchus kisutch, Walbaum). J Exp Biol. 2008;211(Pt 24):3879-88.

16. Carlisle DB, Denton EJ. On the metamorphosis of the visual pigments of Anguilla anguilla (L.). J Mar Biol Assoc U K. 1959;38(1):97-102.

17. Nakamura Y, Mori K, Saitoh K, Oshima K, Mekuchi M, Sugaya T, Shigenobu Y, Ojima N, Muta S, Fujiwara A, et al. Evolutionary changes of multiple visual pigment genes in the complete genome of Pacific bluefin tuna. Proc Natl Acad Sci U S A. 2013;110(27):11061-6.

18. Loew ER, McFarland WN, Margulies D. Developmental changes in the visual pigments of the yellowfin tuna, Thunnus albacares. Mar Fresh Behav Physiol. 2002;35:235-46

19. Lagman D, Ocampo Daza D, Widmark J, Abalo XM, Sundstrom G, Larhammar D. The vertebrate ancestral repertoire of visual opsins, transducin alpha subunits and oxytocin/vasopressin receptors was established by duplication of their shared genomic region in the two rounds of early vertebrate genome duplications. BMC Evol Biol. 2013;13:238.

20. Rennison DJ, Owens GL, Taylor JS. Opsin gene duplication and divergence in ray-finned fish. Mol Phylogenet Evol. 2012;62(3):986-1008.

21. Gojobori J, Innan H. Potential of fish opsin gene duplications to evolve new adaptive functions. Trends Genet. 2009;25(5):198-202

22. Stuart JA, Birge RR. Characterization of the primary photochemical events in bacteriorhodopsin and rhodopsin. Biomembranes: A Multi-Volume Treatise. 1996:2:33-139.

23. Venkatesh B, Ning Y, Brenner S. Late changes in spliceosomal introns define clades in vertebrate evolution. Proc Natl Acad Sci U S A. 1999:96(18):10267-71.

24. Mano H, Kojima D, Fukada Y. Exo-rhodopsin: a novel rhodopsin expressed in the zebrafish pineal gland. Brain Res Mol Brain Res. 1999;73(1-2):110-8.

25. Chen W-J, Bonillo C, Lecointre G. Repeatability of clades as a criterion of reliability: a case study for molecular phylogeny of Acanthomorpha (Teleostei) with larger number of taxa. Mol Phylogenet Evol. 2003;26(2):262-88.

26. Morrow JM, Lazic S, Chang BS. A novel rhodopsin-like gene expressed in zebrafish retina. Vis Neurosci. 2011;28(4):325-35.

27. Pointer MA, Carvalho LS, Cowing JA, Bowmaker JK, Hunt DM. The visual pigments of a deep-sea teleost, the pearl eye Scopelarchus analis. J Exp Biol. 2007;210(Pt 16):2829-35.

28. Zhang $\mathrm{H}$, Futami $\mathrm{K}$, Yamada $\mathrm{Y}$, Horie $\mathrm{N}$, Okamura A, Utoh T, Mikawa N, Tanaka S, Okamoto N, Oka HP. Isolation of freshwater and deep-sea type opsin genes from the common Japanese conger. J Fish Biol. 2002; 61(2):313-24.
29. Archer S, Hope A, Partridge JC. The molecular basis for the green-blue sensitivity shift in the rod visual pigments of the European eel. Proc R Soc Lond B. 1995;262(1365):289-95.

30. Nelson JS: Fishes of the world: Wiley; 2006.

31. Tsukamoto K, Chow S, Otake T, Kurogi H, Mochioka N, Miller MJ, Aoyama J, Kimura S, Watanabe S, Yoshinaga T, et al. Oceanic spawning ecology of freshwater eels in the western North Pacific. Nat Commun. 2011;2:179.

32. Chow S, Kurogi H, Mochioka N, Kaji S, Okazaki M, Tsukamoto K. Discovery of mature freshwater eels in the open ocean. Fish Sci. 2009;75(1):257-9.

33. Righton D, Westerberg $H$, Feunteun E, Okland F, Gargan P, Amilhat E, Metcalfe J, Lobon-Cervia J, Sjoberg N, Simon J, et al. Empirical observations of the spawning migration of European eels: the long and dangerous road to the Sargasso Sea. Sci Adv. 2016:2(10):e1501694.

34. Broughton RE, Betancur RR, Li C, Arratia G, Orti G. Multi-locus phylogenetic analysis reveals the pattern and tempo of bony fish evolution. PLoS Curr. 2013;5

35. Inoue JG, Miya M, Tsukamoto K, Nishida M. A mitogenomic perspective on the basal teleostean phylogeny: resolving higher-level relationships with longer DNA sequences. Mol Phylogenet Evol. 2001;20(2):275-85.

36. Zhang H, Futami K, Horie N, Okamura A, Utoh T, Mikawa N, Yamada Y, Tanaka S, Okamoto N. Molecular cloning of fresh water and deep-sea rod opsin genes from Japanese eel Anguilla japonica and expressional analyses during sexual maturation. FEBS Lett. 2000;469(1):39-43.

37. Hope AJ, Partridge JC, Hayes PK. Switch in rod opsin gene expression in the European eel, Anguilla anguilla (L). Proc R Soc Lond B. 1998; 265(1399):869-74

38. Wood P, Partridge JC. Opsin substitution induced in retinal rods of the ee (Anguilla anguilla (L.)): a model for G-protein-linked receptors. Proc Royal Soc Lond B. 1993:254(1341):227-32.

39. Morrow JM, Lazic S, Dixon Fox M, Kuo C, Schott RK, AGE D, Santini F, Tropepe V, Chang BS. a second visual rhodopsin gene, rh1-2, is expressed in zebrafish photoreceptors and found in other ray-finned fishes. J Exp Biol. 2017:220(Pt 2):294-303.

40. Minamoto T, Shimizu I. Molecular mechanism of visual adaptation in fish. Jpn J Ichthyol. 2005;52(2):91-106.

41. Li J, Bian C, Hu Y, Mu X, Shen X, Ravi V, Kuznetsova IS, Sun Y, You X, Qiu Y, et al. A chromosome-level genome assembly of the Asian arowana, Scleropages formosus. Sci Data. 2016;3:160105.

42. Tanaka H. Progression in artificial seedling production of Japanese eel Anguilla japonica. Fish Sci. 2015;81(1):11-9.

43. Asahida T, Kobayashi T, Saitoh K, Nakayama I. Tissue preservation and total DNA extraction form fish stored at ambient temperature using buffers containing high concentration of urea. Fish Sci. 1996;62(5):727-30.

44. Langmead B, Salzberg SL. Fast gapped-read alignment with bowtie 2. Nat Methods. 2012;9(4):357-9.

45. Boetzer M, Henkel CV, Jansen HJ, Butler D, Pirovano W. Scaffolding preassembled contigs using SSPACE. Bioinformatics. 2011;27(4):578-9.

46. Boetzer M, Pirovano W. Toward almost closed genomes with GapFiller. Genome Biol. 2012;13(6):R56

47. Zhu D, Song W, Yang K, Cao X, Gul Y, Wang W. Flow cytometric determination of genome size for eight commercially important fish species in China. In Vitro Cell Dev Biol Anim. 2012:48(8):507-17.

48. Marcais $G$, Kingsford C. A fast, lock-free approach for efficient parallel counting of occurrences of $k$-mers. Bioinformatics. 2011;27(6):764-70.

49. Kai W, Nomura K, Fujiwara A, Nakamura Y, Yasuike M, Ojima N, Masaoka T, Ozaki A, Kazeto Y, Gen K, et al. A ddRAD-based genetic map and its integration with the genome assembly of Japanese eel (Anguilla japonica) provides insights into genome evolution after the teleost-specific genome duplication. BMC Genomics. 2014;15:233.

50. Stanke M, Diekhans M, Baertsch R, Haussler D. Using native and syntenically mapped CDNA alignments to improve de novo gene finding. Bioinformatics. 2008;24(5):637-44

51. Kim D, Pertea G, Trapnell C, Pimentel H, Kelley R, Salzberg SL. TopHat2: accurate alignment of transcriptomes in the presence of insertions, deletions and gene fusions. Genome Biol. 2013;14(4):R36.

52. Trapnell C, Williams BA, Pertea G, Mortazavi A, Kwan G, van Baren MJ, Salzberg SL, Wold BJ, Pachter L. Transcript assembly and quantification by RNA-Seq reveals unannotated transcripts and isoform switching during cell differentiation. Nat Biotechnol. 2010;28(5):511-5.

53. Yates A, Akanni W, Amode MR, Barrell D, Billis K, Carvalho-Silva D, Cummins C, Clapham P, Fitzgerald S, Gil L, et al. Ensembl 2016. Nucleic Acids Res. 2016;44(D1):D710-6. 
54. Altschul SF, Madden TL, Schaffer AA, Zhang J, Zhang Z, Miller W, Lipman DJ. Gapped BLAST and PSI-BLAST: a new generation of protein database search programs. Nucleic Acids Res. 1997;25(17):3389-402.

55. Jones P, Binns D, Chang HY, Fraser M, Li W, McAnulla C, McWilliam H, Maslen J, Mitchell A, Nuka G, et al. InterProScan 5: genome-scale protein function classification. Bioinformatics. 2014;30(9):1236-40.

56. Rondeau EB, Minkley DR, Leong JS, Messmer AM, Jantzen JR, von Schalburg KR, Lemon C, Bird NH, Koop BF. The genome and linkage map of the northern pike (Esox lucius): conserved synteny revealed between the salmonid sister group and the Neoteleostei. PLoS One. 2014;9(7):e102089.

57. Haas BJ, Papanicolaou A, Yassour M, Grabherr M, Blood PD, Bowden J, Couger MB, Eccles D, Li B, Lieber M, et al. De novo transcript sequence reconstruction from RNA-seq using the trinity platform for reference generation and analysis. Nat Protoc. 2013;8(8):1494-512.

58. Li L, Stoeckert CJ Jr, Roos DS. OrthoMCL: identification of ortholog groups for eukaryotic genomes. Genome Res. 2003;13(9):2178-89.

59. Slater GS, Birney E. Automated generation of heuristics for biological sequence comparison. BMC Bioinformatics. 2005;6:31.

60. Henkel CV, Burgerhout E, de Wijze DL, Dirks RP, Minegishi Y, Jansen HJ, Spaink HP, Dufour S, Weltzien FA, Tsukamoto K, et al. Primitive duplicate Hox clusters in the European eel's genome. PLoS One. 2012;7(2):e32231.

61. Kurtz S, Phillippy A, Delcher AL, Smoot M, Shumway M, Antonescu C, Salzberg SL. Versatile and open software for comparing large genomes. Genome Biol. 2004;5(2):R12

62. Katoh K, Asimenos G, Toh H. Multiple alignment of DNA sequences with MAFFT. Methods Mol Biol. 2009;537:39-64.

63. Saitoh K, Sado T, Doosey MH, Bart HL Jr, Inoue JG, Nishida M, Mayden RL, Miya M. Evidence from mitochondrial genomics supports the lower Mesozoic of South Asia as the time and place of basal divergence of cypriniform fishes (Actinopterygii: Ostariophysi). Zool J Linnean Soc. 2011; 161(3):633-62.

64. Tamura K, Stecher G, Peterson D, Filipski A, Kumar S. MEGA6: molecular evolutionary genetics analysis version 6.0. Mol Biol Evol. 2013;30(12):2725-9.

65. Yang Z. PAML 4: phylogenetic analysis by maximum likelihood. Mol Biol Evol. 2007;24(8):1586-91.

66. Cao Y, Kim KS, Ha JH, Hasegawa M. Model dependence of the phylogenetic inference: relationship among carnivores, Perissodactyls and Cetartiodactyls as inferred from mitochondrial genome sequences. Genes Genet Syst. 1999; 74(5):211-7.

67. Hasegawa $\mathrm{M}$, Kishino $\mathrm{H}$. Accuracies of the simple methods for estimating the bootstrap probability of a maximum-likelihood tree. Mol Biol Evol. 1994; 11(1):142-5.

68. Shimodaira H, Hasegawa M. CONSEL: for assessing the confidence of phylogenetic tree selection. Bioinformatics. 2001;17(12):1246-7.

69. Steinway SN, Dannenfelser R, Laucius CD, Hayes JE, Nayak S. JCoDA: a tool for detecting evolutionary selection. BMC Bioinformatics. 2010;11:284.

70. Yokoyama S, Tada T, Zhang H, Britt L. Elucidation of phenotypic adaptations: molecular analyses of dim-light vision proteins in vertebrates. Proc Natl Acad Sci U S A. 2008;105(36):13480-5.

71. Jansen HJ, Liem M, Jong-Raadsen SA, Dufour S, Weltzien FA, Swinkels W, Koelewijn A, Palstra AP, Pelster B, Spaink HP, et al. Rapid de novo assembly of the European eel genome from nanopore sequencing reads. Sci Rep. 2017;7(1):7213.

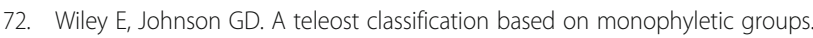
Origin and phylogenetic interrelationships of teleosts. 2010;1:123-82.

73. Chen JN, Lopez JA, Lavoue S, Miya M, Chen WJ. Phylogeny of the Elopomorpha (Teleostei): evidence from six nuclear and mitochondrial markers. Mol Phylogenet Evol. 2014;70:152-61.

74. Nakamura Y. A mathematical model for gene evolution after whole genome duplication. In: ArXiv e-prints vol. 1702;2017

75. Inoue JG, Sato Y, Sinclair R, Tsukamoto K, Nishida M. Rapid genome reshaping by multiple-gene loss after whole-genome duplication in teleost fish suggested by mathematical modeling. Proc Natl Acad Sci U S A. 2015; 112(48):14918-23.

76. Lynch M, Conery JS. The evolutionary fate and consequences of duplicate genes. Science. 2000;290(5494):1151-5.

77. Lafont AG, Rousseau K, Tomkiewicz J, Dufour S. Three nuclear and two membrane estrogen receptors in basal teleosts, Anguilla sp.: identification, evolutionary history and differential expression regulation. Gen Comp Endocrinol. 2016:235:177-91.
78. Robertson FM, Gundappa MK, Grammes F, Hvidsten TR, Redmond AK, Lien S, Martin SAM, Holland PWH, Sandve SR, Macqueen DJ. Lineage-specific rediploidization is a mechanism to explain time-lags between genome duplication and evolutionary diversification. Genome Biol. 2017;18(1):111.

79. Christensen KA, Davidson WS. Autopolyploidy genome duplication preserves other ancient genome duplications in Atlantic salmon (Salmo Salar). PLoS One. 2017;12(2):e0173053.

80. Saitoh K. Mitotic and meiotic analyses of the 'large race' of Cobitis Striata, a polyploid spined loach of hybrid origin. Folia Biol (Krakow). 2003;51(Suppl):101-5.

81. Morini M, Penaranda DS, Vilchez MC, Tveiten H, Lafont AG, Dufour S, Perez $L$, Asturiano JF. The expression of nuclear and membrane estrogen receptors in the European eel throughout spermatogenesis. Comp Biochem Physiol A Mol Integr Physiol. 2017;203:91-9.

82. Ogino $Y$, Kuraku S, Ishibashi H, Miyakawa H, Sumiya E, Miyagawa S, Matsubara H, Yamada G, Baker ME, Iguchi T. Neofunctionalization of androgen receptor by gain-of-function mutations in teleost fish lineage. Mol Biol Evol. 2016;33(1):228-44.

83. Douard V, Brunet F, Boussau B, Ahrens-Fath I, Vlaeminck-Guillem V, Haendler $B$, Laudet $V$, Guiguen $Y$. The fate of the duplicated androgen receptor in fishes: a late neofunctionalization event? BMC Evol Biol. 2008;8:336.

84. Guo B, Gan X, He S. Hox genes of the Japanese eel Anguilla japonica and Hox cluster evolution in teleosts. J Exp Zool B Mol Dev Evol. 2010;314(2):135-47.

85. Dornburg A, Friedman M, Near TJ. phylogenetic analysis of molecular and morphological data highlights uncertainty in the relationships of fossil and living species of Elopomorpha (Actinopterygii: Teleostei). Mol Phylogenet Evol. 2015:89:205-18.

86. Santini F, Kong X, Sorenson L, Carnevale G, Mehta RS, Alfaro ME. A multilocus molecular timescale for the origin and diversification of eels (order: Anguilliformes). Mol Phylogenet Evol. 2013;69(3):884-94.

87. Watanabe HC, Mori Y, Tada T, Yokoyama S, Yamato T. Molecular mechanism of long-range synergetic color tuning between multiple amino acid residues in conger rhodopsin. Biophysics (Oxf). 2010;6:67-8.

88. Inoue JG, Miya M, Miller MJ, Sado T, Hanel R, Hatooka K, Aoyama J, Minegishi Y, Nishida M, Tsukamoto K. Deep-ocean origin of the freshwater eels. Biol Lett. 2010;6(3):363-6.

\section{Submit your next manuscript to BioMed Central and we will help you at every step:}

- We accept pre-submission inquiries

- Our selector tool helps you to find the most relevant journal

- We provide round the clock customer support

- Convenient online submission

- Thorough peer review

- Inclusion in PubMed and all major indexing services

- Maximum visibility for your research

Submit your manuscript at www.biomedcentral.com/submit
C) Biomed Central 